\title{
Des lasers \\ à impulsions femtosecondes pour mesurer les fréquences
}

Anne Amy-Klein (anne.amy-klein@univ-paris13.fr)

Laboratoire de physique des lasers, Université Paris 13, 93430 Villetaneuse

Les lasers à impulsions

de durée femtoseconde

ont longtemps été développés

uniquement pour leurs

caractéristiques temporelles.

Cependant, leurs propriétés

spectrales sont aussi extra-

ordinaires, car leur spectre

est constitué d'un peigne de

fréquences optiques extrê-

mement régulier, qui peut

être exploité pour réaliser

des mesures de fréquences

de très haute précision.

Cette découverte a valu

à T. W. Hänsch et J. L. Hall

le prix Nobel de physique 2005.

Dans cet article, nous expliquons

les bases de cette technique

de mesure de fréquences et

en décrivons quelques-unes

des applications les plus

passionnantes.
Les mesures de fréquences permettent actuellement d'effectuer les expériences les plus sensibles de la physique, car l'unité de temps-fréquence est l'unité réalisée, de loin, avec la meilleure incertitude : de l'ordre de $10^{-16}$ en valeur relative. Les applications sont très variées et intéressent autant le grand public (géopositionnement par satellites, analyses en temps réel...) que les chercheurs de pointe (variation temporelle des constantes fondamentales de la physique, tests de relativité générale...). Cependant, pour atteindre de telles précisions, les dispositifs de mesures doivent également être caractérisés par des incertitudes extrêmement basses. Pour des radiofréquences ou des micro-ondes, divers composants électroniques sont disponibles et la chaîne de mesure est relativement simple. Pour des fréquences plus élevées, et en particulier pour les fréquences optiques, les composants électroniques ne sont pas assez rapides, et, jusqu'aux années 2000, la mesure précise de fréquences nécessitait des dispositifs extrêmement complexes de " chaînes de fréquences ", qui en limitaient très fortement le développement.

Depuis une dizaine d'années, les chaînes de mesure sont simplement constituées d'un laser à impulsions de durée femtoseconde (fs). Un seul instrument permet donc de comparer des fréquences allant du domaine des radiofréquences jusqu'à l'ultraviolet. Cela a véritablement révolutionné la métrologie des fréquences et a donné lieu en 2005 à l'attribution du prix Nobel de physique à T. W. Hänsch et J. L. Hall, pour "leur contribution au développement de la spectroscopie laser de précision et, en particulier, de la technique du peigne de fréquences optiques".

\section{Propriétés spectrales du laser femtoseconde}

Les lasers à impulsions émettent à intervalles réguliers des impulsions de lumière de formes identiques, comme illustré par la figure 1a. Le spectre se déduit par simple transformation de Fourier. Il est constitué d'un peigne de fréquences très large de plusieurs dizaines de térahertz $(\mathrm{THz})$ (l'inverse de la durée de l'impulsion), pour lequel la distance entre modes est donnée par la fréquence de répétition $f_{\mathrm{r}}$ du laser, qui est l'inverse du temps séparant deux impulsions successives (fig. 1b). Il a été vérifié expérimentalement que les modes étaient parfaitement équidistants, même aux deux extrémités du peigne, et ceci, à un niveau d'au moins $10^{-17}$ en valeur relative. C'est l'interférence constructive de tous ces modes, correspondant à une très large gamme de fréquences, qui permet d'obtenir des impulsions très courtes. Cependant, la fréquence d'un mode n'est pas directement un multiple entier de la fréquence de répétition : le spectre est décalé de $\mathrm{f}_{0}$ à l'origine. Ainsi, chaque mode a pour fréquence $f_{p}=\left(p \times f_{r}\right)+f_{0}$, où $p$ est un entier qui définit le mode, et les fréquences $\mathrm{f}_{\mathrm{r}}$ et $\mathrm{f}_{0}$ sont des paramètres caractéristiques du laser. Le décalage $\mathrm{f}_{0}$ a pour origine physique la différence entre les vitesses de phase et de groupe de l'onde laser. Dans le domaine temporel, il induit un déphasage $\Delta \varphi$ à chaque impulsion entre la porteuse et l'enveloppe de l'impulsion.

Concrètement, la fréquence de répétition $\mathrm{f}_{\mathrm{r}}$ est de l'ordre de $100 \mathrm{MHz}$ à $1 \mathrm{GHz}$, le décalage $\mathrm{f}_{0}$ est situé entre 0 et $\mathrm{f}_{\mathrm{r}}$, tandis que le laser femtoseconde émet dans le domaine 


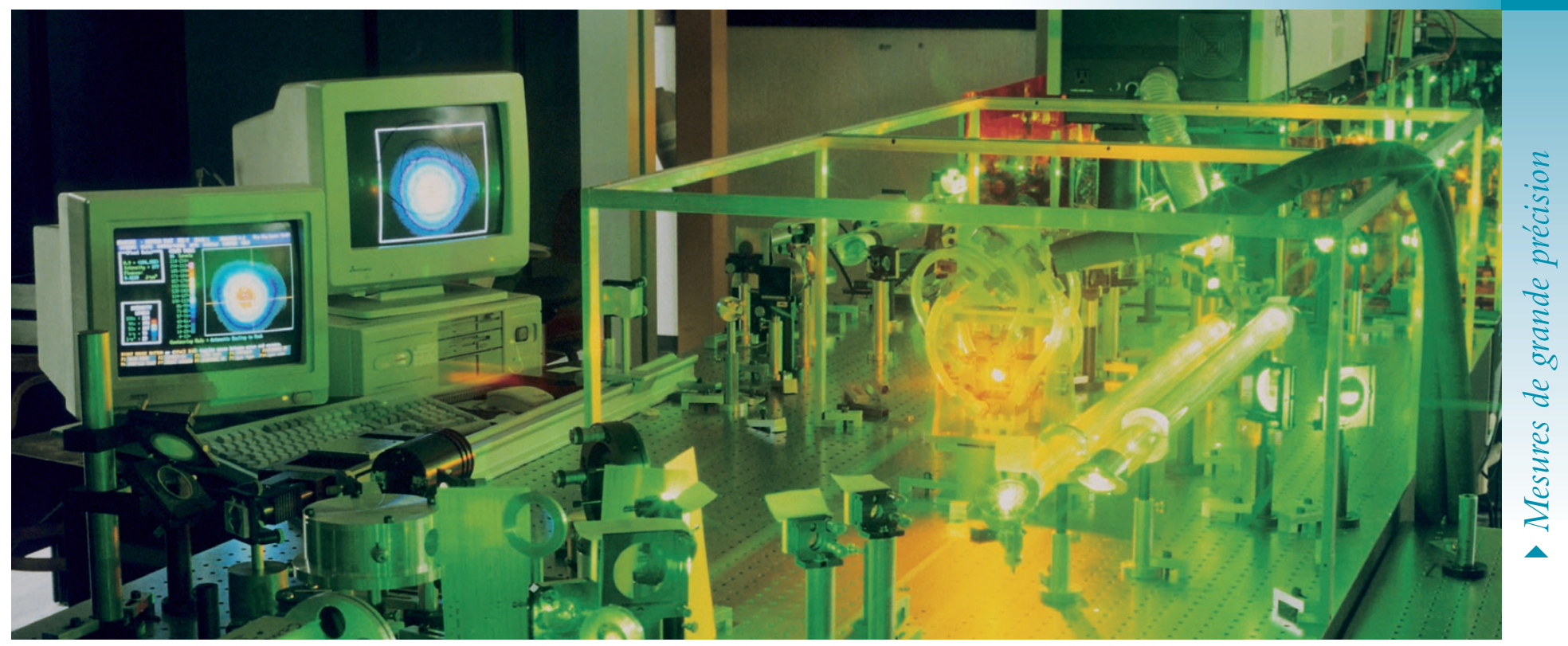

Chaîne laser femtoseconde 35 TW (Laboratoire d'optique appliquée, Palaiseau). o cNRS Photothèque / ENSTA / LOCHEGnIES José.

optique : autour de $800 \mathrm{~nm}$ pour les lasers utilisant un cristal de saphir dopé au titane, ou de $1500 \mathrm{~nm}$ pour les lasers à fibres dopées en erbium. Les modes du laser femtoseconde ont donc des fréquences de l'ordre de $c /\left(8 \times 10^{-7} \mathrm{~m}\right)$ ou $c /\left(1,5 \times 10^{-6} \mathrm{~m}\right)$ (où c est la vitesse de la lumière dans le vide), c'est-à-dire quelques $10^{14} \mathrm{~Hz}$. D'après la relation $\mathrm{f}_{\mathrm{p}}=\left(\mathrm{p} \times \mathrm{f}_{\mathrm{r}}\right)+\mathrm{f}_{0}$, on en déduit que les modes du peigne correspondent à des valeurs très élevées de $\mathrm{p}$, de l'ordre de $10^{5}$.

Le spectre du laser femtoseconde est donc caractérisé par deux paramètres seulement : le taux de répétition du laser, $\mathrm{f}_{\mathrm{r}}$, et le décalage global du peigne de fréquences, $\mathrm{f}_{0}$, tous les deux situés dans le domaine radiofréquence. Le peigne de fréquences du laser femtoseconde peut alors être utilisé comme une "règle" de fréquences. On mesure le battement $\Delta$ de la fréquence fà mesurer avec le mode le plus proche du peigne, par exemple le mode $\mathrm{p}: \Delta=\mathrm{f}-\mathrm{f}_{\mathrm{p}}$, puis on en déduit $f=\Delta+\left(p \times f_{r}\right)+f_{0}$. On a ainsi ramené la mesure de la fréquence optique $\mathrm{f}$ à celles de l'entier $\mathrm{p}$ et des radiofréquences $\mathrm{f}_{\mathrm{r}}$ et $\mathrm{f}_{0}$, inférieures de quatre à cinq ordres de grandeur. L'entier $\mathrm{p}$ est facilement obtenu à partir d'une première mesure grossière de $f$, ou bien on l'estime à partir de deux mesures successives obtenues avec deux valeurs différentes de $f_{r}$. La mesure d'une fréquence optique dépend alors simplement de la détermination des deux paramètres $f_{r}$ et $f_{0}$. Comme ces derniers dépendent, entre autres, de la longueur L de la cavité laser, ils doivent être contrôlés très précisément, comme expliqué plus loin, par rapport à une référence qui reproduit l'unité de fréquence. L'incertitude sur la mesure de fréquence dépend uniquement de l'incertitude sur la détermination ou le contrôle de ces deux paramètres, comme nous le verrons plus en détail en troisième partie.

Le laser femtoseconde permet également de comparer deux fréquences optiques différentes: $\mathrm{f}_{1}=\Delta_{1}+\left(\mathrm{p}_{1} \times \mathrm{f}_{\mathrm{r}}\right)+\mathrm{f}_{0}$ et $f_{2}=\Delta_{2}+\left(p_{2} \times f_{r}\right)+f_{0}$, en éliminant la contribution de $f_{r}$, et en mesurant par ailleurs $\mathrm{f}_{0}$. Enfin, le doublage de fréquences offre la possibilité d'étendre cette technique de mesure au proche ultraviolet.

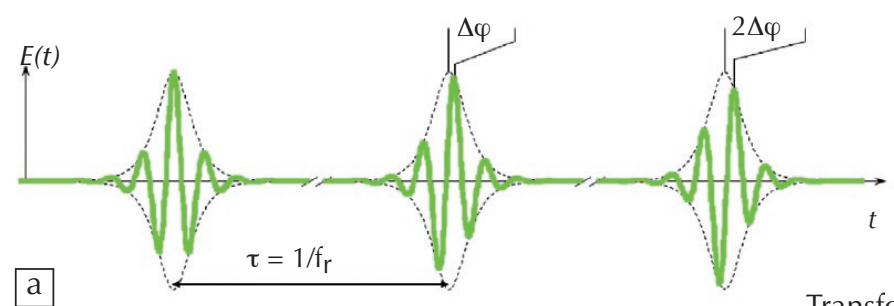

Transformée de Fourier

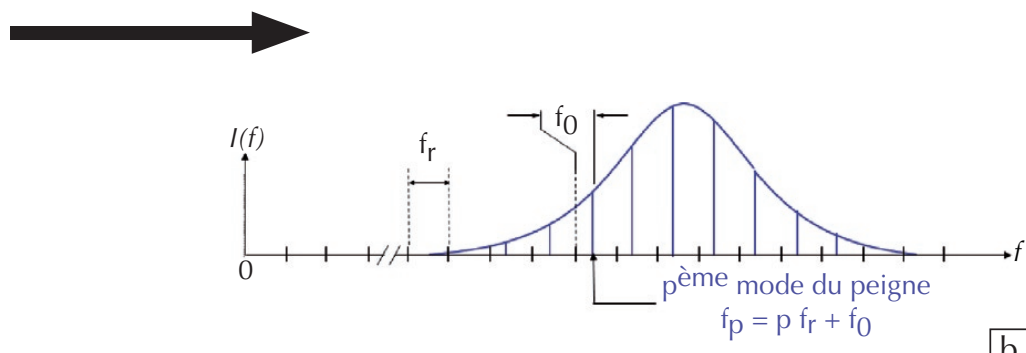

1. Représentation de l'émission d'un laser femtoseconde en fonction du temps et de la fréquence. (a) Train d'impulsions émis par un laser à modes bloqués ; le déphasage $\Delta \varphi$ correspond au décalage du maximum du champ, par rapport au centre de l'impulsion, d'une impulsion à l'autre. (b) Spectre du laser à impulsions, qui correspond à la transformée de Fourier de ce champ ; les graduations donnent les harmoniques (ou fréquences multiples) de $\mathrm{f}_{\mathrm{r}}$. 


\section{Contrôle de la fréquence des modes du laser femtoseconde}

Nous allons maintenant expliquer comment détecter et contrôler les deux paramètres du peigne. Le lecteur moins intéressé par ces détails techniques, pourra passer directement à la partie suivante.

La fréquence de répétition est un paramètre simple à mesurer et à contrôler. Il suffit d'envoyer une partie du faisceau laser femtoseconde sur une photodiode rapide pour détecter le "battement " entre les divers modes de l'impulsion, et ainsi mesurer $\mathrm{f}_{\mathrm{r}}$. Cette fréquence de répétition est directement reliée à la longueur $\mathrm{L}$ de la cavité du laser par la relation : $\mathrm{f}_{\mathrm{r}}=\mathrm{v}_{\mathrm{g}} / \mathrm{L}$, où $\mathrm{v}_{\mathrm{g}}$ est la vitesse de groupe de l'impulsion. La fréquence $f_{r}$ varie au cours du temps, car la longueur optique de la cavité laser fluctue et dérive en fonction, entre autres, de la température. Il est donc nécessaire, soit de mesurer $f_{r}$ en temps réel, soit de stabiliser sa valeur à une valeur de référence fixée. Dans ce dernier cas, il suffit d'agir sur la longueur L de la cavité. On peut monter, par exemple, un des miroirs de la cavité laser sur une céramique piezoélectrique dont l'épaisseur varie en fonction de la tension appliquée, ce qui permet de déplacer le miroir très finement.

La détermination de $\mathrm{f}_{0}$ est beaucoup plus délicate, et la technique couramment utilisée nécessite un dispositif particulier pour élargir l'impulsion sur plus d'une octave. Cela a été rendu possible depuis les années 2000 par l'apparition des fibres microstructurées (fig. 2), qui ont la propriété de propager avec la même vitesse les différents modes de l'impulsion. Dans ces fibres de forme très spécifique, la dispersion de vitesse de groupe associée au guide est importante, ce qui permet de compenser la dispersion de la silice, et ainsi d'ajuster le zéro de dispersion autour de la longueur d'onde d'utilisation. Avec cette annulation de la dispersion, et un cœur très petit, ces fibres microstructurées sont sujettes à de très forts effets non linéaires. On obtient ainsi un élargissement spectaculaire des impulsions sur plus d'une octave.

Pour mesurer le décalage $\mathrm{f}_{0}$, la méthode couramment utilisée consiste à engendrer les modes de fréquences doubles de la partie basse fréquence du peigne, par génération de second harmonique dans un cristal non linéaire. Si le peigne s'étend sur plus d'une octave, la partie basse fréquence du spectre généré se recouvre alors avec la partie
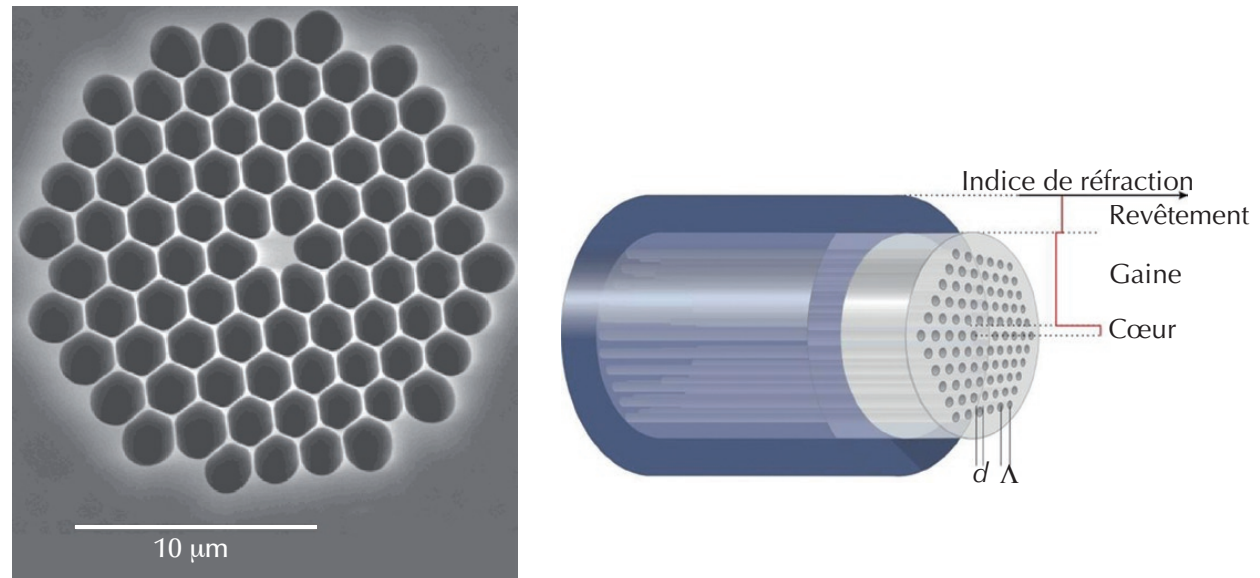

2. Fibre dite " à cristaux photoniques "

(à gauche : image extraite de www.blazephotonics.com/ ; à droite : image extraite de www.thorlabs.de/)
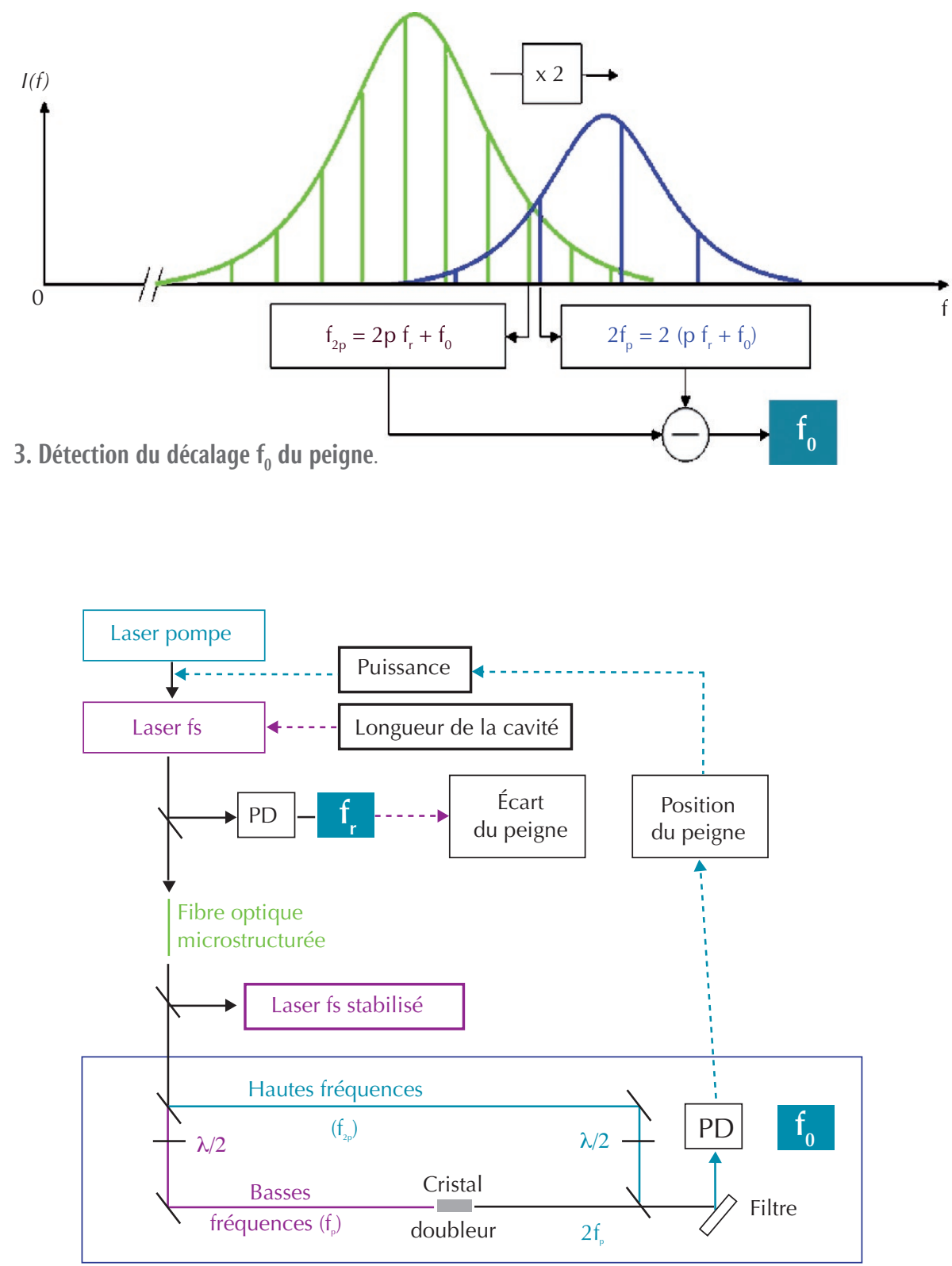

4. Montage « autoréférencé » pour le contrôle des paramètres du peigne (PD : photodétecteur). 
haute fréquence du spectre initial. On peut alors trouver plusieurs valeurs de $\mathrm{p}$ pour lesquelles coexistent le mode harmonique de fréquence $2 f_{p}$ et le mode de fréquence $f_{2 p} d u$ peigne initial. Ces modes $2 f_{p}$ et $f_{2 p}$ interferent et on peut mesurer le signal de battement à la fréquence $2 f_{p}-f_{2 p}$, qui correspond exactement au décalage $\mathrm{f}_{0}$, quelle que soit la valeur de $\mathrm{p}$ (fig. 3) :

$2 f_{p}-f_{2 p}=2\left(p f_{r}+f_{0}\right)-\left(2 p f_{r}+f_{0}\right)=f_{0}$.

Ensuite, le contrôle de $\mathrm{f}_{0}$ s'effectue en agissant, par exemple, sur la puissance du laser de pompe du laser femtoseconde. En effet, cela modifie l'indice du milieu non linéaire responsable de l'effet laser, et donc la vitesse de groupe, dont dépend $\mathrm{f}_{0}$. Le montage global est illustré sur la figure 4.

\section{Mesures absolues de fréquence et mesures de très haute sensibilité}

Revenons aux mesures de fréquences. Pour être exactes, les mesures des deux paramètres $\mathrm{f}_{\mathrm{r}}$ et $\mathrm{f}_{0}$ (et de $\Delta$ ) doivent être calibrées par rapport à un étalon de fréquence, qui réalise le mieux possible l'unité de temps-fréquence. Celle-ci est définie, depuis 1968, à partir de la transition entre les deux sous-niveaux hyperfins du niveau fondamental de l'atome de césium. Leur différence d'énergie ne doit pas varier a priori au cours du temps, et constitue donc une référence absolue. La fréquence de la transition a été fixée arbitrairement à $9192631770 \mathrm{~Hz}$ exactement, ce qui définit le hertz $(\mathrm{Hz})$, et par conséquent la seconde. L'étalon primaire de temps-fréquence est une horloge qui réalise cette unité.

La solution la plus simple consiste à se référer au signal du GPS : on peut ainsi obtenir une incertitude de $3 \times 10^{-10}$ sur $10 \mathrm{~s}$ et une exactitude de l'ordre de $3 \times 10^{-12}$. Pour des performances environ cent fois meilleures, il faut utiliser une horloge au rubidium ou au césium, également commerciale. Enfin, pour obtenir les meilleures performances, il est nécessaire de se référer aux étalons primaires développés dans les laboratoires nationaux de métrologie. On peut alors réaliser des mesures de fréquences optiques, avec des incertitudes de l'ordre de $10^{-14}$ à $10^{-16}$ en valeur relative.

Au-delà des applications purement métrologiques, la possibilité de réaliser des mesures de fréquences avec une précision très élevée permet d'explorer les limites de la physique fondamentale. Ainsi, la transition 1s-2s de l'atome d'hydrogène a été mesurée en 1999 avec une précision relative de $1,8 \times 10^{-14}$, soit une amélioration d'un facteur 20 par rapport aux mesures précédentes. En combinant cette mesure avec celle d'une autre transition de l'hydrogène, on peut en déduire la constante de Rydberg et les corrections radiatives dues à l'électrodynamique quantique. Cela permet de tester très précisément les modèles de cette théorie ou bien, si ces modèles sont supposés valides, de mesurer le rayon de charge du proton, pour lequel coexistent actuellement deux mesures contradictoires.

Ces mesures de fréquence sont également sensibles à une éventuelle variation des constantes fondamentales avec le temps. En effet, les fréquences de transitions de résonance atomiques ou moléculaires s'expriment en fonction de diverses constantes fondamentales, telles que les masses de l'électron et du proton, la constante de Rydberg, la constante de structure fine... Le laser femtoseconde permet de comparer, et donc de déterminer, le rapport de deux fréquences de transitions atomiques ou moléculaires. Ce rapport s'exprime également en fonction des constantes fondamentales et est susceptible de varier au cours du temps, car les théories qui se situent au-delà du Modèle standard, notamment celles des supercordes et de " grande unification ", prévoient toutes que les constantes usuelles ne sont pas stables dans le temps et l'espace. Cela doit se traduire par une variation des fréquences avec le temps, qui pourrait être de l'ordre de $10^{-15}$ à $10^{-18}$ par an, en valeur relative.

Plusieurs groupes ont récemment interprété leurs mesures de fréquences pour donner une limite supérieure à une éventuelle variation de la constante de structure fine, de l'ordre de $10^{-15}$ par an en valeur relative. Le caractère complémentaire des diverses comparaisons permet d'extraire la variation de chaque constante séparément. Il est aussi essentiel qu'une éventuelle variation temporelle des constantes fondamentales puisse être validée par plusieurs expériences. Les expériences de comparaison de fréquences devraient bientôt pouvoir être en mesure de contraindre les théories. Il s'agit ici d'une perspective passionnante, offerte par ce nouveau dispositif.

\section{Bilan et perspectives}

Depuis une dizaine d'années, les chercheurs ont découvert que les lasers à impulsions femtosecondes se présentent comme des peignes de fréquences optiques, permettant de comparer des fréquences différant de plusieurs ordres de grandeur. Ce dispositif, simple et compact, rend accessible à un grand nombre de laboratoires une palette d'expériences réservées jusque-là aux laboratoires nationaux de métrologie. Le champ d'applications est très large : comparaison d'horloges atomiques ou moléculaires, calibration de spectres astrophysiques, spectroscopie de Fourier avec des peignes de fréquence...

Dans ce dernier développement, l'utilisation d'une source laser femtoseconde permet de sonder l'absorption d'une molécule, simultanément pour toutes les fréquences des modes du peigne. C'est une technique très rapide et très sensible, qui est donc susceptible de se développer au niveau industriel pour l'analyse spectroscopique en temps réel : détection de traces, contrôle de procédés, analyse biomédicale...

L'ensemble de ces applications, imprévisibles il y a une douzaine d'années, témoigne des potentialités exceptionnelles du laser, qui continue de susciter de nouveaux thèmes de recherche ou développement, cinquante ans après sa découverte.

\section{Pour en savoir plus}

- Site web du Prof. Hänsch, prix Nobel de physique 2005 : www.mpq.mpg.de/ haensch/comb/index.html

- Site web du Prof. Hall, prix Nobel de physique 2005 : www.nist.gov/public_affairs/releases/frequency_ combs.ffm

- Le laser, ouvrage coordonné par N. Treps et F. Bretenaker, EDP Sciences (2010).

- «Impulsions lumineuses ultracourtes pour la métrologie de fréquences", Images de la physique 2005. CNRS.

- Femtosecond optical frequency comb technology, J. Ye et S.T. Cundiff, eds., Springer, New York (2005).

- J.R. Hall et al., IEEE J. of Quantum Electronics 37 (2001) 1482-1492; R. Holzwarth et al., id., 1493-1501. L. Hollberg et al., id., 1502-1513.

- S.T. Cundiff et al., Review of Scientific Intruments 72 (2001) 3749 . 